\title{
Effect of Silica Nanoparticles on the Local Segmental Dynamics in Poly(vinyl acetate)
}

\author{
R. B. Bogoslovov ${ }^{\dagger}$ C. M. Roland,$* \dagger$ A. R. Ellis, ${ }^{\dagger}$ A. M. Randall, $\stackrel{\ddagger}{\ddagger}$ and C. G. Robertson ${ }^{\ddagger}$ \\ Chemistry Division, Code 6120, Naval Research Laboratory, Washington, D.C. 20375-5342, and \\ Bridgestone Americas, Center for Research and Technology, 1200 Firestone Parkway, Akron, \\ Ohio 44317-0001
}

Received October 25, 2007; Revised Manuscript Received December 10, 2007

\begin{abstract}
The effect of nanosized silica particles on the properties of poly(vinyl acetate) (PVAc) was investigated for a range of silica concentrations encompassing the percolation threshold. The quantity of polymer adsorbed to the particles ("bound rubber") increased systematically with silica content and was roughly equal to the quantity shielded from shear stresses ("occluded rubber"). This bound and occluded polymer attained a level of $\sim 12 \%$ at a silica volume content of $28 \%$; nevertheless, the glass transition properties of the PVAc, including the glass transition temperature, local segmental relaxation function and relaxation times, and the changes in thermal expansion coefficient and heat capacity at $T_{\mathrm{g}}$, were unaffected by the interfacial material. That is, there is no indication that the local segmental dynamics of the chains adjacent to silica particles differ from the motions of the bulk chains. Interestingly, the volume sensitivity of the segmental dynamics, as determined from the scaling exponent $\gamma$ in the relation $T_{\mathrm{g}} \sim V_{\mathrm{g}}^{-\gamma}$ in which $V_{\mathrm{g}}$ is the specific volume at the glass transition, becomes stronger with increasing silica concentration. Moreover, this dependence of $\gamma$ increases abruptly at the filler percolation threshold. The implication of this result and possible directions for new research are considered.
\end{abstract}

\section{Introduction}

Nanoparticle reinforcement of rubber is almost as old as the use of rubber itself. The incorporation of hard particles into soft polymers usually serves two purposes: reducing cost and improving performance. The main property enhancements are stiffness and strength, although better processability (e.g., shape retention), abrasion resistance, fluid impermeability, fire resistance, and electrical conductivity can also be realized. Optimal reinforcement requires a continuum of nanoparticle contacts, with the minimum concentration ("percolation threshold") for a flocculated network depending on particle size, shape, and interaction with the polymer. The presence of a network confers nonlinearity to the mechanical response, since the particle contacts can be broken by strain. This leads to the well-known Payne effect: the marked reduction in the dynamic storage modulus and appearance of a maximum in the loss modulus at strain amplitudes in the range $0.1-10 \% .^{1-5}$ Although the dynamic modulus of filled rubber exhibits a weak dependence on strain amplitude even at low particle concentrations, a strong nonlinearity accompanies the continuity of the particle contacts effected by high concentrations. This jamming-unjamming transition $^{6}$ is seen generally in colloidal suspensions and other thixotropic fluids. Recent work has shown that the disruption of the particle network transpires at a characteristic strain energy, independent of the nature of the particles or their concentration. ${ }^{7-9}$ This isoenergetic feature of the unjamming is also observed in sheared gels. ${ }^{10}$

Of interest herein is the effect of nanoparticle reinforcement on the local segmental dynamics. These motions underlie many important properties of polymers ${ }^{11}$ and also function as the precursor to the larger length scale chain motions associated with processing and flow. ${ }^{12}$ Constraints from nanoparticles may affect segmental mobility, at least of chains near the particle

\footnotetext{
Naval Research Laboratory.

$\doteqdot$ Bridgestone Americas.
}

surface, and it has been proposed that immobilization of such interfacial chains induces glassy behavior at temperatures above the bulk $T_{\mathrm{g}} \cdot{ }^{13-16}$ Relatedly, there have been various reports of increases in the glass transition temperature, $T_{\mathrm{g}}$, due to the presence of reinforcing fillers: ${ }^{17-22}$ however, other investigators found no effect of carbon black, silica, and other particles on $T_{\mathrm{g}}{ }^{23,24}$ The effect of nanoconfinement on the polymer dynamics, seen in thin films and porous structures, ${ }^{25-27}$ may have an influence for layered fillers such as clay platelets. ${ }^{28,29}$ An obvious direct consequence of filler is that less material participates in the glass transition, suppressing the changes in the temperature coefficients of the modulus, enthalpy, volume, etc., occurring on heating through $T_{\mathrm{g}}{ }^{30}$ Temperature changes also influence the extent of the interactions among the nanoparticles due to changes in their mutual proximity and their Brownian motion. ${ }^{31}$ This confers additional complexity to the local segmental response of the polymer in the vicinity of the glass transition.

In this work we investigate poly(vinyl acetate) (PVAc) mixed with silica nanoparticles. PVAc has a $T_{\mathrm{g}}$ above ambient temperature. While this precludes application as a general purpose elastomer, it makes it convenient to measure properties both above and below $T_{\mathrm{g}}$. This is important for our PVT experiments, which rely on mercury as a confining fluid and thus cannot be carried out at low temperatures ( $\mathrm{Hg}$ crystallizes at $\sim-39^{\circ} \mathrm{C}$ ). Silica has found increasing applications as a filler, for example in the tire industry to reduce the rolling resistance of the tread rubber, ${ }^{32-34}$ and a number of studies of silica reinforcement have appeared. ${ }^{4,22,35-43}$ The silica used herein consists of spherical particles with surfaces treated to reduce the polarity and thereby provide stronger interaction with the polymer segments. The concentration of the silica was varied to determine the effect on certain properties, in particular those associated with the local segmental dynamics.

\section{Experimental Section}

The poly(vinyl acetate) (Sigma-Aldrich) had a weight-average molecular weight $=167 \mathrm{~kg} / \mathrm{mol}$ and a polydispersity $=2.01$. The 


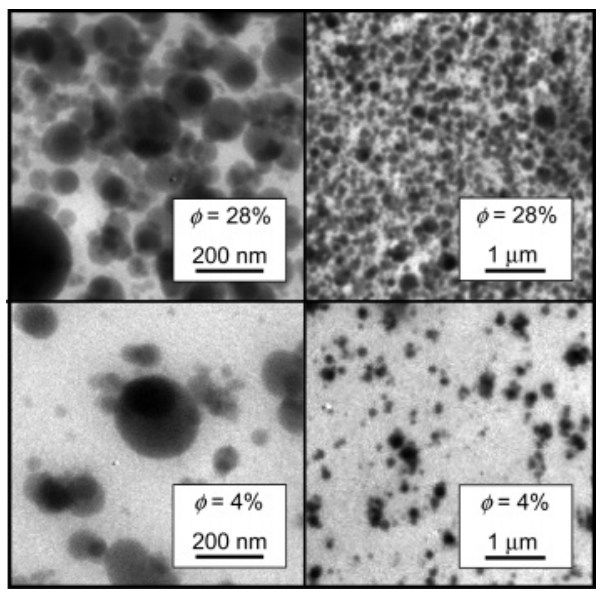

Figure 1. TEM images of PVAc containing 0.28 (top) and 0.04 (bottom) volume fraction of silica. The dispersion of the particles results in transparency of the samples.

filler was Nan-O-Sil amorphous colloidal silica from Energy Strategy Associates, Inc. These are spherical particles with an average diameter of $\sim 100 \mathrm{~nm}$ and surface treated with tetrasulfidosilane. The silica particles were incorporated into the polymer using a Brabender internal mixer at $70{ }^{\circ} \mathrm{C}$ followed by mixing on a two-roll mill. The final compounds were transparent, consistent with good dispersion of the silica. Figure 1 shows representative transmission electron micrographs. The filler volume fraction was in the range from $\phi=0$ to 0.282 ( $\leq 0.70$ parts of silica per polymer by weight), as verified by thermogravimetric analysis of the final compounds. Bound rubber was determined as the unextracted PVAc after immersion in an excess of toluene for 3 days at $23{ }^{\circ} \mathrm{C}$.

Modulated differential scanning calorimetry (MDSC) was carried out using a TA Instruments Q100, calibrated with a sapphire standard. Samples, thoroughly dried prior to measurements, were cooled in the calorimeter from 80 to $-10{ }^{\circ} \mathrm{C}$ at $1{ }^{\circ} \mathrm{C} / \mathrm{min}$. The modulation was $\pm 1 \mathrm{deg}$, with a $60 \mathrm{~s}$ period. Viscosities were measured at $70{ }^{\circ} \mathrm{C}$ in a Mooney viscometer using the small rotor. Dynamic shear measurements employed a TA Instruments ARES rheometer, using a cone and plate geometry $(15 \mathrm{~mm}$ diameter, 0.1 rad angle), with data obtained at $70{ }^{\circ} \mathrm{C}$ and a frequency $=1 \mathrm{rad} / \mathrm{s}$. A Novocontrol Alpha analyzer was used for dielectric relaxation measurements, in combination with a Delta Design model 9023 oven. The pressure and temperature dependences of the volume were measured using a Gnomix instrument, ${ }^{44}$ in which the sample is immersed in mercury contained in a flexible bellows. At various fixed pressures over the range from 10 to $160 \mathrm{MPa}$, the volume change was measured as temperature was lowered from ca. $40 \mathrm{deg}$ above $T_{\mathrm{g}}$ to $-10{ }^{\circ} \mathrm{C}$ at a rate of $0.5^{\circ} \mathrm{C} / \mathrm{min}$. To determine the bulk modulus in the rubbery state, the change in volume was also measured isothermally as a function of pressure, with the latter increased from 10 to $80 \mathrm{MPa}$ at a rate of $4 \mathrm{MPa} / \mathrm{min}$. From the specific gravity at ambient $T$ and $P$ measured by the buoyancy method, these volume changes were converted to specific volume, $V$.

\section{Results}

Filler-Polymer Interaction. Surface treatment of the silica with tetrasulfidosilane reduces the polarity of the silica, which reduces the particle-particle attraction strength and also, by making the filler surface more compatible with the polymer, enhances filler-polymer interactions. Enhanced interactions between silica and PVAc can make the interfacial polymer insoluble in a good solvent and potentially reduces the segmental mobility of these chains. The degree and consequences of this immobilization have been subjects of various studies. ${ }^{13-24,41-43}$ Medalia $^{45}$ determined that the shielding of the occluded polymer layer from mechanical stresses increases the effective concentra-

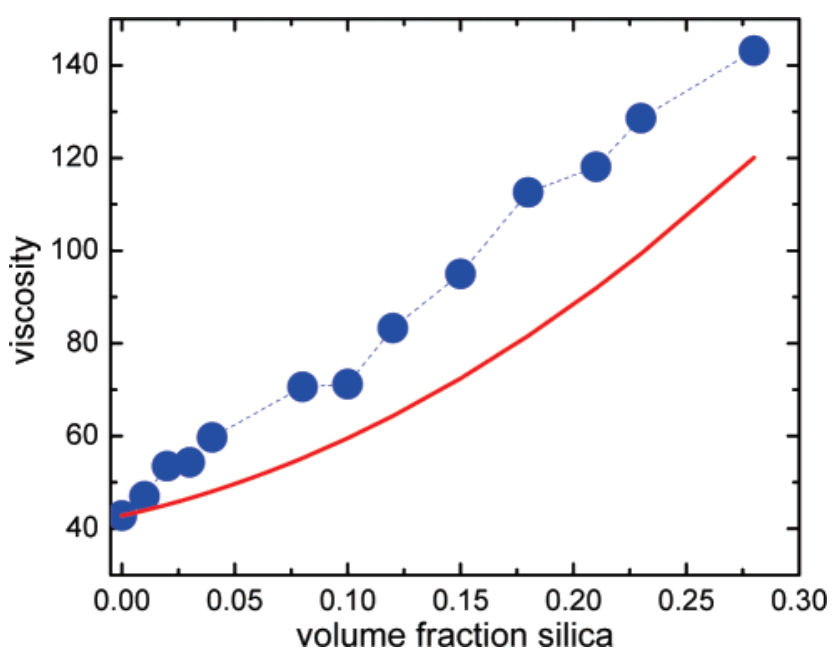

Figure 2. Viscosity in Mooney units at $70{ }^{\circ} \mathrm{C}$ (circles) vs silica concentration. The solid line is the viscosity calculated from eq 1 , while the broken line was calculated assuming a portion of the polymer is occluded, increasing the effective $\phi$.

tion of the filler. In Figure 2 are the flow viscosities, $\eta$, of the rubbers, showing the expected increase with the filler content. An equation to describe the effect of suspended, rigid spheres on the viscosity of a fluid is the Einstein equation, modified by Guth and Gold for higher particle concentrations ${ }^{46}$

$$
\eta(\phi)=\eta(0)\left(1+2.5 \phi+14.1 \phi^{2}\right)
$$

where $\eta(0)$ is the viscosity of the neat fluid. Equation 1 assumes no interaction between the fluid and particles, so that the viscosity depends on the concentration of particles but not on their size or shape. Equation 1 underestimates $\eta$ for the filled PVAc (solid line in Figure 2).

To improve the fit to experimental data, one can include higher order terms in eq 1 or use alternative expressions, ${ }^{47}$ for example the equation of Oliver and Ward for the viscosity of a dispersion of spheres ${ }^{48}$

$$
\eta(\phi)=\eta(0)(1-a \phi)
$$

where $a$ is a constant. However, the problem with these expressions is neglect of the polymer in the interstitial regions at the particle interface. A method to account for this occluded polymer was suggested by Medalia: ${ }^{45}$ eq 1 is fit to the data by letting $\phi$ be an adjustable parameter, representing the effective concentration, that is, the sum of the silica plus occluded polymer. As seen in Figure 3, the quantity of occluded rubber, calculated as the difference between the filler concentration reproducing the $\eta(\phi)$ data in Figure 2 and the actual silica concentration, increases with silica content, comprising as much as $10 \%$ of the polymer at large $\phi$. At high $\phi$ the quantity of bound rubber (chains firmly attached to the silica particles) exceeds the amount of occluded rubber.

Filler-Filler Interaction. The dynamic storage modulus, $G^{\prime}$, is plotted vs strain amplitude in Figure 4 for PVAc with varying concentration of silica. At low strain there is a plateau in $G^{\prime}$, but the modulus decreases with strain for the higher silica levels. This is the well-known Payne effect, ${ }^{1}$ reflecting mechanical disruption of the flocculated particle network. The concentration of particles necessary for this agglomeration can be determined from a plot (Figure 5) of $G^{\prime}$ at low strain amplitude vs $\phi$. Beyond $\sim 11 \%$ silica there is an increase in the slope, demarcating the development of long-range continuity of particle contacts. The connectivity of the particles enhances their contribution to the 


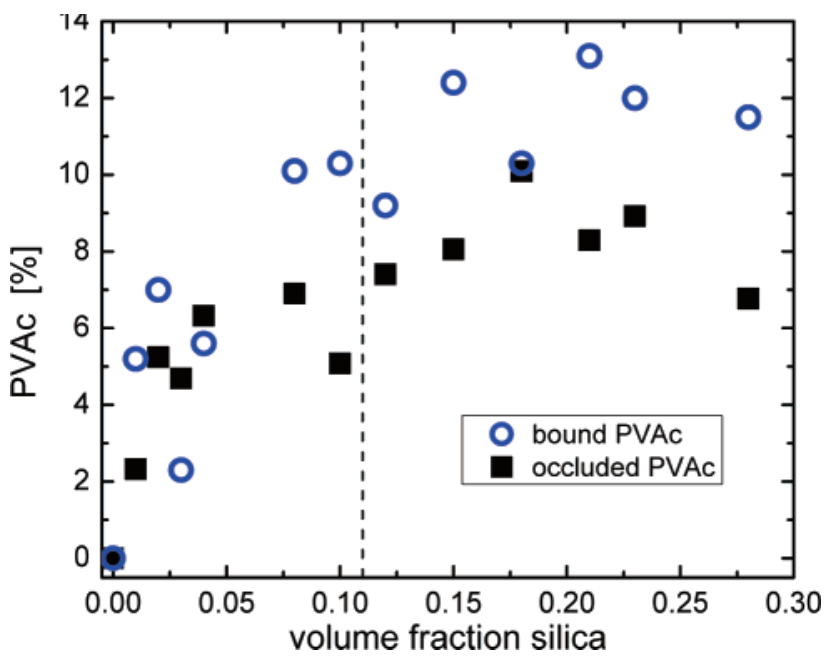

Figure 3. Bound (open circles) and occluded (filled squares) PVAc as a function of silica content. The vertical dashed line denotes the minimum filler concentration for formation of an agglomerated network.

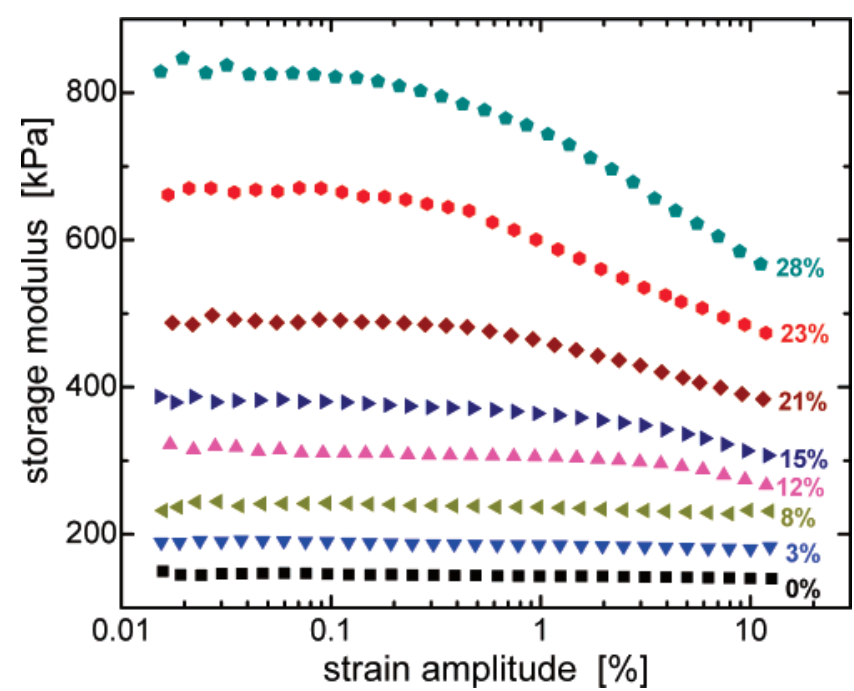

Figure 4. Dynamic storage modulus as a function of shear strain for PVAc at $70{ }^{\circ} \mathrm{C}$ with varying silica levels (as indicated by volume).

mechanical response and can give rise to other effects, such as higher thermal or electrical conductivity. ${ }^{49,50}$

Local Segmental Dynamics. The glass transition in polymers denotes the onset of local segmental motions (correlated conformational transitions of a few backbone bonds), reflected inter alia as a change in the variation of the heat capacity, $C_{p}$, with temperature. The calorimetric $T_{\mathrm{g}}$ measured for the samples was unaffected by the presence of the silica concentration; $T_{\mathrm{g}}$ $=40.4 \pm 0.7^{\circ} \mathrm{C}$ independent of $\phi$. (Note this value from MDSC is about 2 deg higher than obtained by conventional DSC at the same rate of temperature change.) The literature concerning the effect of reinforcing nanofillers on $T_{\mathrm{g}}$ is mixed, ${ }^{13-24}$ but we have recently shown ${ }^{51}$ that some confusion arises from the use of mechanical determinations of $T_{\mathrm{g}}$, specifically the loss tangent $(\tan \delta$ ). The filler-induced stiffening of the rubber (e.g., Figures 2 and 4) can skew the shape of the loss tangent, unaccompanied by any significant change in the segmental dynamics underlying the glass transition per se; this may result in the false impression of a higher $T_{\mathrm{g}}$.

The calorimetric $T_{\mathrm{g}}$ is identified with the temperature of an abrupt increase in $C_{p}$ due to the onset of liquidlike mobility with increasing $T$. In Figure 6, we plot (filled triangles) the magnitude of this step change in heat capacity, $\Delta C_{P}$, vs $\phi$. As

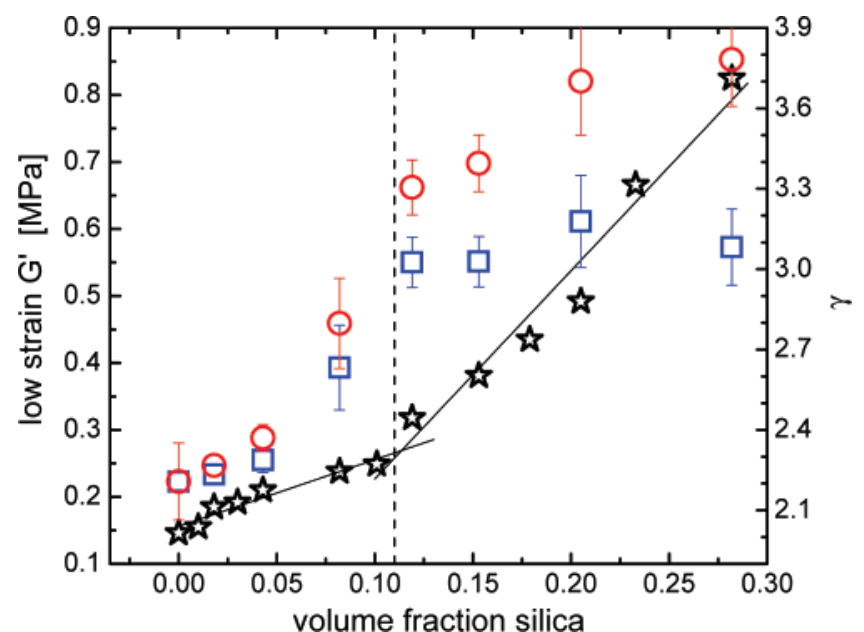

Figure 5. Variation with silica concentration of the storage modulus at strain amplitudes $<0.1 \%$ (stars; left ordinate scale) and of the scaling exponent (right ordinate scale; squares calculated using the specific volume of the compound and circles using $V$ corrected for the PVAc content). The vertical dashed line denotes the minimum filler concentration for formation on a silica network.

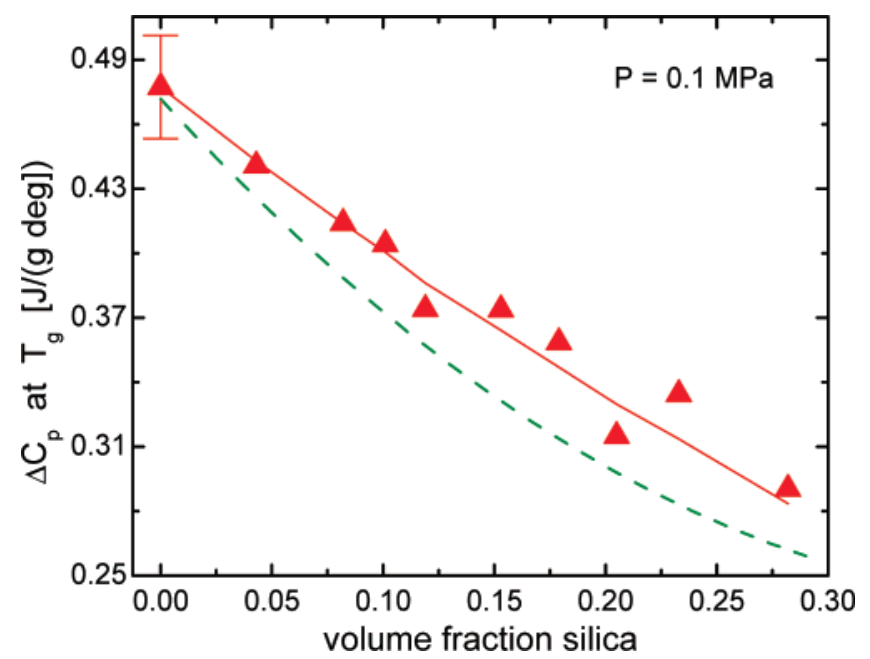

Figure 6. Difference in heat capacity between the glassy and rubbery states at $T_{\mathrm{g}}$ (solid triangles), along with the $\Delta C_{P}$ calculated assuming no contribution from the silica (solid line) and assuming no contribution from the silica or the occluded rubber (dashed line).

the silica concentration increases, $\Delta C_{P}$ decreases, as expected since $T_{\mathrm{g}}$ only involves the polymer. In fact, the entire reduction in $\Delta C_{P}$ can be accounted for solely by consideration of the amount of polymer present; this is indicated by the solid line through the data, representing $\Delta C_{P}(\phi)=\Delta C_{P}(0)\left[(1-\phi) \rho_{\mathrm{R}}\right] /$ $\left[\phi \rho_{\mathrm{f}}+(1-\phi) \rho_{\mathrm{R}}\right]$, where $\rho_{\mathrm{R}}(=1.18 \mathrm{~g} / \mathrm{mL})$ and $\rho_{\mathrm{f}}(=2.1 \mathrm{~g} / \mathrm{mL})$ are the respective mass densities of the PVAc and silica. Thus, all PVAc segments participate in the glass transition, including the substantial number of occluded chains. This is seen in Figure 6 , wherein the heat capacity increment calculated after subtraction of the occluded chains (dashed curve) underestimates the measured values of $\Delta C_{P}$.

Above $T_{\mathrm{g}}$ the variation in the heat capacity itself with $\phi$ follows the same trend. In fact, the magnitude of $C_{P}$ for the silica is sufficiently small, $C_{P} \sim 0.7 \mathrm{~J} /\left(\mathrm{g}{ }^{\circ} \mathrm{C}\right)$ at $\mathrm{RT},{ }^{52}$ that its contribution can be ignored given the experimental scatter of the measurement of the heat capacity.

There is a similar step increase in the thermal expansivity as temperature is increased through $T_{\mathrm{g}}$. To quantify this, we carried out PVT measurements on the rubbers, with representative results for three compositions $(\phi=0,0.082$, and 0.282 ) shown 

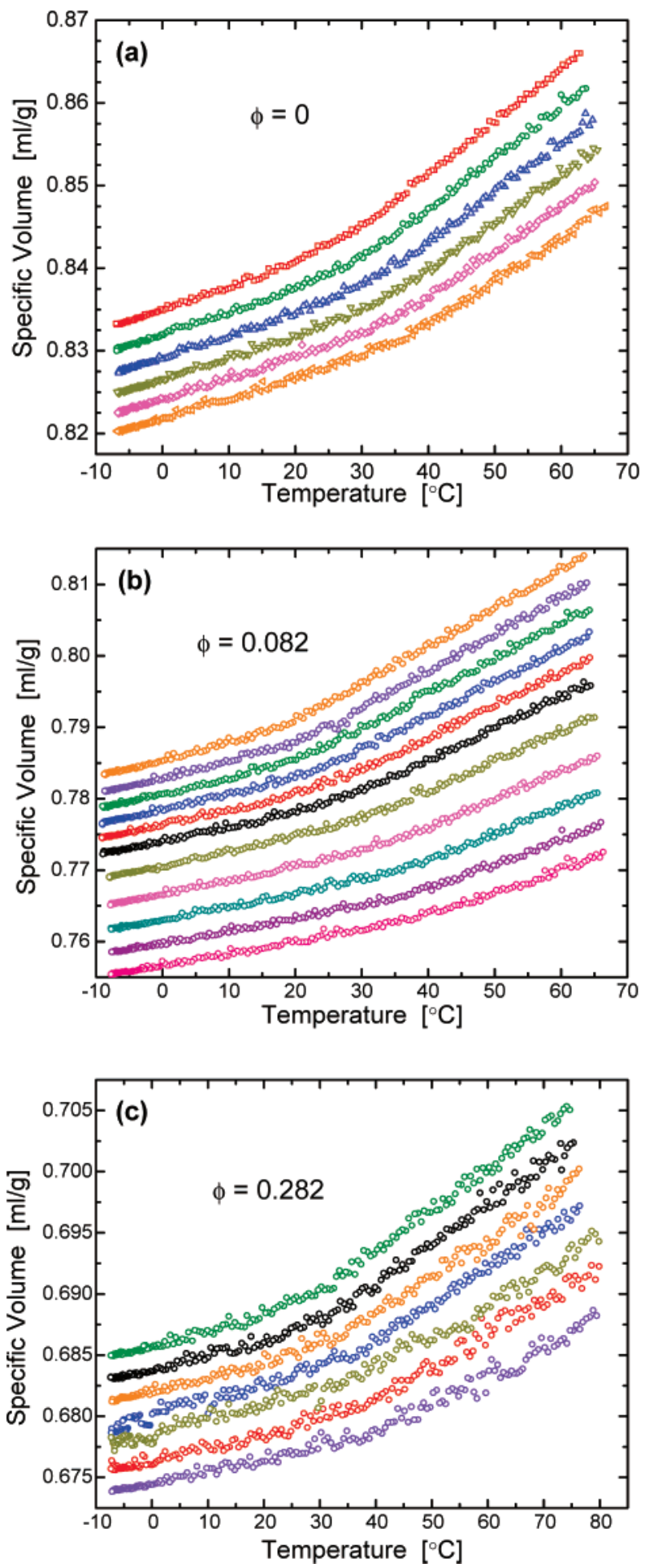

Figure 7. Specific volume as a function of temperature with pressure increasing from the top to the lower curves: (a) neat PVAc (10-60 $\mathrm{MPa}, 10 \mathrm{MPa}$ increments); (b) $8.2 \%$ silica (10-60 MPa, $10 \mathrm{MPa}$ increments; 60-160 MPa, $20 \mathrm{MPa}$ increments); (c) $28.2 \%$ silica (10$60 \mathrm{MPa}, 10 \mathrm{MPa}$ increments and $80 \mathrm{MPa}$ ).

in Figure 7. Over the limited temperature range, the $V(T)$ data in both the rubbery and glassy states could be fit to a linear equation, with the isobaric thermal expansion coefficient obtained as $\alpha_{P}=(\mathrm{d} V / \mathrm{d} T) V^{-1}$. Results extrapolated to $T=T_{\mathrm{g}}$ (at $P=10 \mathrm{MPa}$, the lowest measurable pressure of the PVT instrument) are displayed in Figure 8 for all samples. Generally, the thermal expansion of filled rubber is dominated by the polymer. ${ }^{53}$ Since silica is an amorphous glass having a network

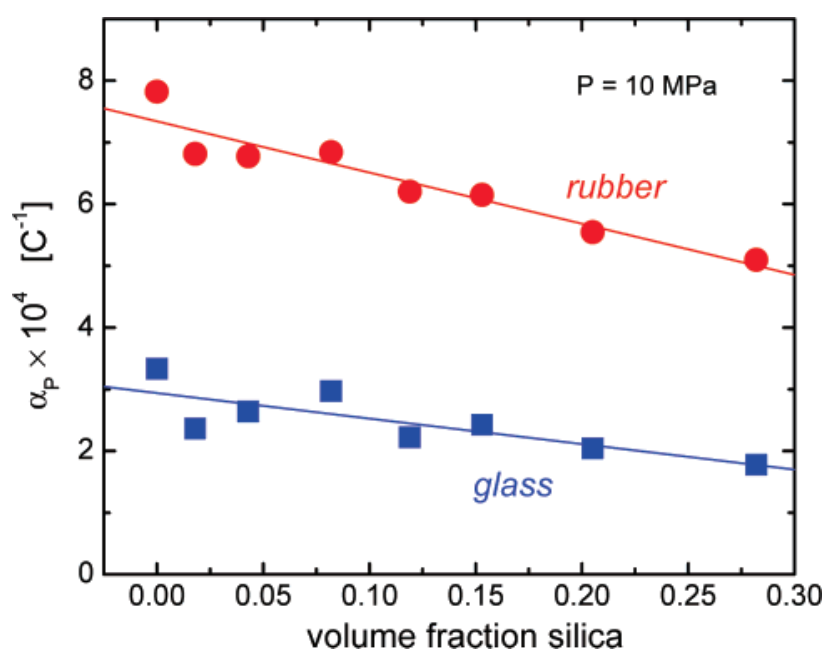

Figure 8. Thermal expansion coefficient for glassy (squares) and rubbery (circles) states, with the solid lines representing $\alpha_{P}$ calculated assuming no contribution from the silica.

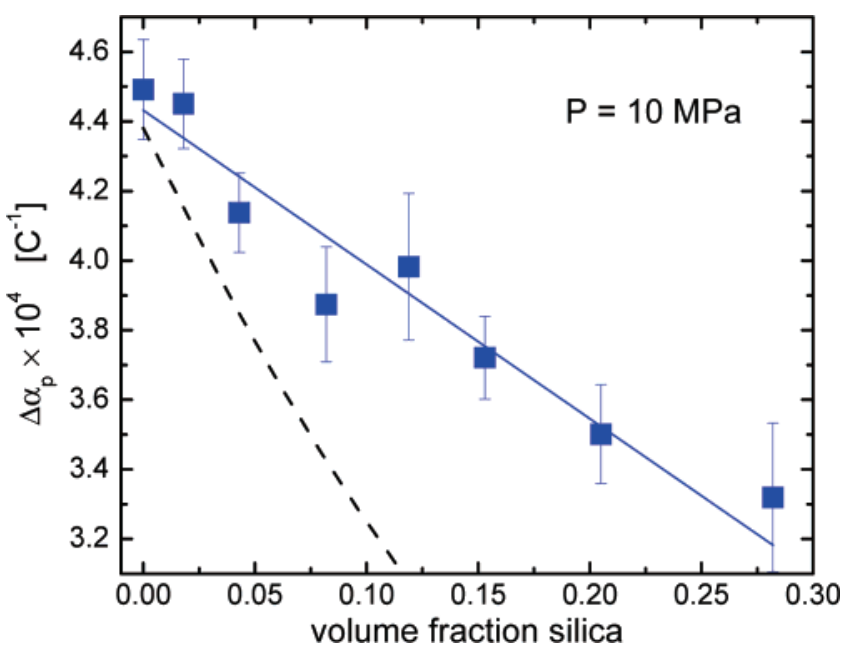

Figure 9. Difference in thermal expansivity of glass and rubber at $T_{\mathrm{g}}$. The solid line assumes that all PVAc contributes to the transition strength; i.e., $\Delta \alpha_{P}(\phi)=(1-\phi) \Delta \alpha_{P}(0)$. The dashed line assumes the occluded rubber does not participate in the glass transition.

structure, its thermal expansivity is very small, $\alpha_{P}=10^{-6}$ per ${ }^{\circ} \mathrm{C} .{ }^{54}$ Thus, we can assume that the volume expansion is due solely to the PVAc, and indeed the equation $\alpha_{P}(\phi)=(1-$ $\phi) \alpha_{P}(0)$ describes accurately the experimental data (solid lines in Figure 8). Taking the difference of the values for the rubbery and glassy states, we obtain $\Delta \alpha_{P}$, the change in the thermal expansion coefficient at $T_{\mathrm{g}}$ (Figure 9). Similar to the heat capacity jump at $T_{\mathrm{g}}$, these data reflect the contribution of all polymer segments (solid line in Figure 9). Subtracting the occluded chains from the polymer concentration leads to calculated $\Delta \alpha_{P}$ (dashed line) that underestimate the measured values.

It is well-established that segmental relaxation times, $\tau_{\alpha}$, are accurately described over broad ranges of thermodynamic conditions by the scaling law

$$
\tau_{\alpha}=f\left(T V^{\gamma}\right)
$$

in which $\gamma$ is a material constant, independent of $T$ and $P$, and $f$ represents some function, unknown a priori. ${ }^{55}$ Together with the fact that $\tau_{\alpha}$ is constant at $T_{\mathrm{g}}$ (that is, as $P$ increases, $T_{\mathrm{g}}$ 


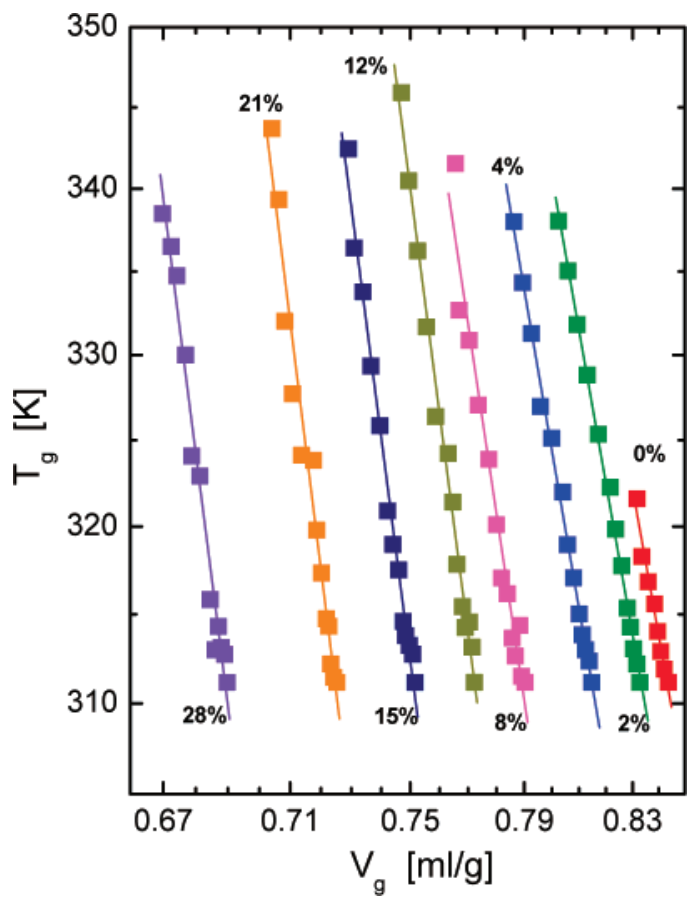

Figure 10. Glass transition temperature vs specific volume at $T_{\mathrm{g}}$, with the points of each curve corresponding to a different pressure and each curve representing a different sample (silica content as indicated). Note that both scales are logarithmic.

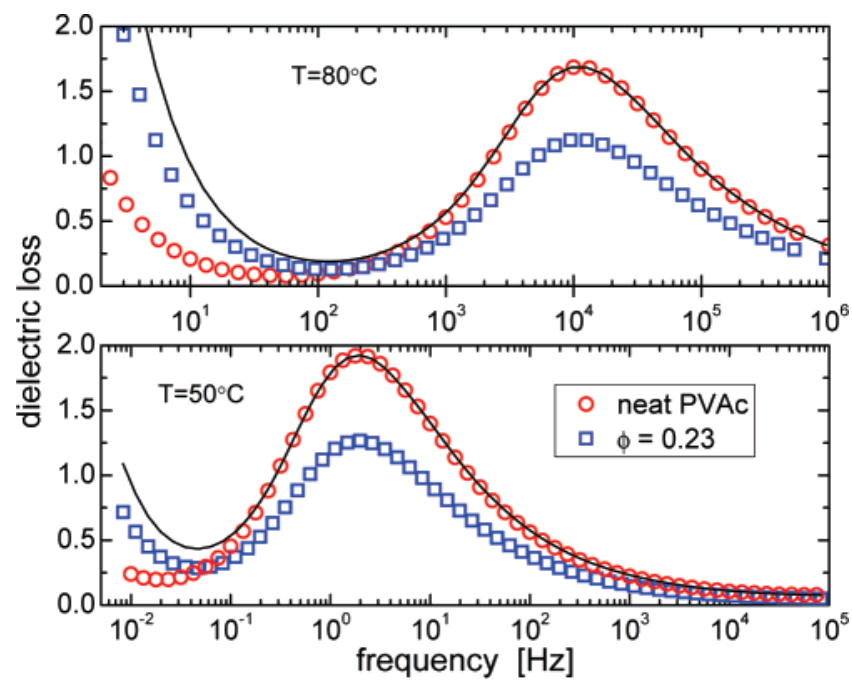

Figure 11. Dispersion in the dielectric loss due to local segmental motion (circles: $\phi=0$; squares: $\phi=0.23$ ) at the indicated temperatures. When divided by 0.66 to normalize for the mass fraction of polymer, the peak for the filled PVAc (solid line) superposes on the peak for neat PVAc. The rise in the dielectric loss toward lower frequencies is due to the conductivity, which becomes more separated at higher $T$.

increases but $\tau_{\alpha}\left(T_{\mathrm{g}}, P\right)$ does not $),{ }^{55}$ this means that the $T_{\mathrm{g}}$ and the volume at the glass transition are related according to

$$
T_{\mathrm{g}} \propto V_{\mathrm{g}}^{-\gamma}
$$

The proportionality of the two quantities means that a doublelogarithmic plot of $T_{\mathrm{g}}$ (in kelvin) vs $V_{\mathrm{g}}$ will have a slope equal to $-\gamma$. The appeal of this analysis is that it enables the dependence of $\tau_{\alpha}$ to be determined without actual relaxation data. Such a plot is shown in Figure 10. The $\gamma$, obtained from fitting eq 4 to the experimental points, increases with $\phi$; in particular, there is a change in the $\gamma(\phi)$ behavior at the silica

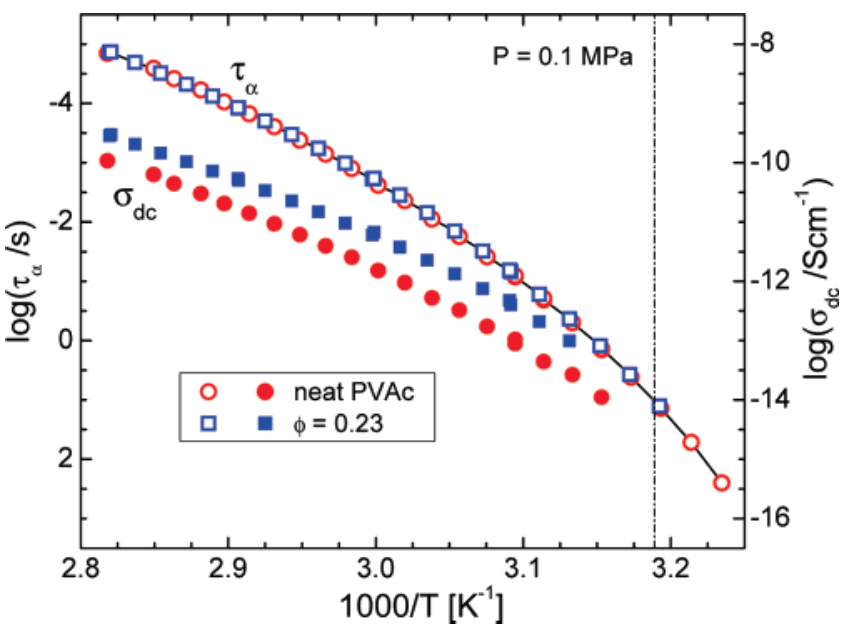

Figure 12. Local segmental relaxations times (open symbols) defined from the inverse of the peak frequency) for unfilled and filled PVAc; the $\tau_{\alpha}(T)$ are seen to be equivalent. The solid line is the fit of eq 5 to the combined data. Also shown is the dc conductivity (filled symbols), which is larger in the PVAc containing silica due to a higher concentration of mobile ions. (Note the ordinate scale for $\tau_{\alpha}$ is reversed to facilitate comparison with $\sigma_{\mathrm{dc}}$.) The vertical dashed line designates the calorimetric $T_{\mathrm{g}}$.

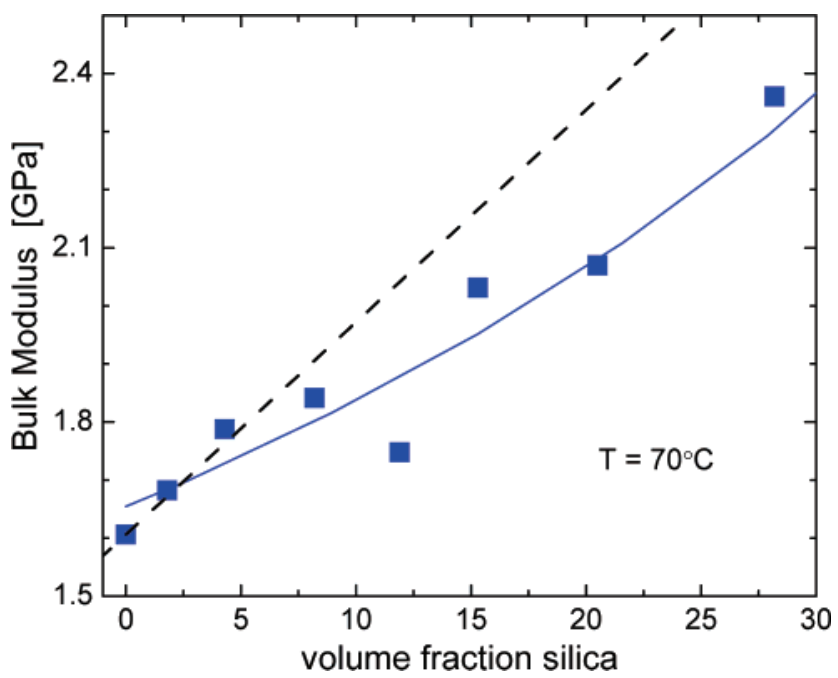

Figure 13. Bulk modulus measured in the rubbery state (squares) along with calculated value (solid line) assuming only the PVAc is compressible. The dashed line assumes the occluded rubber, as well as the silica, is incompressible.

concentration associated with formation of a filler network (Figure 5).

An uncertainty in this analysis is the correct value of $V$ to use in determining $\gamma$-the sample specific volume (squares in Figure 5) or the remainder after subtraction of the silica content (circles in Figure 5). The latter is more realistic; however, the differences in the obtained $\gamma$ are not large and do not change the results qualitatively, as seen in the figure.

Given that the volume dependence of $\tau_{\alpha}$ is affected by the filler, it is of interest to measure directly the segmental relaxation times. We carried out dielectric relaxation measurements at ambient pressure on the neat polymer and PVAc containing $23 \%$ silica. From these data, $\tau_{\alpha}$ for other pressures could be calculated using eq 3 and the equation of state (see below), but we restrict our attention herein to the relaxation behavior at zero pressure. In Figure 11 are shown the local segmental relaxation peaks at 50 and $80{ }^{\circ} \mathrm{C}$ for the two materials. When the spectra for the filled PVAc are divided by the mass fraction of polymer (= 0.66 for the volumetric concentration of $\phi=0.23$ ), at both 
Table 1. Equation-of-State Parameters (Eq 6) for the PVAc/Silica Compounds

\begin{tabular}{|c|c|c|c|c|c|c|}
\hline$\phi(\%)$ & $V^{a}(\mathrm{~mL} / \mathrm{g})$ & $V_{0}(\mathrm{~mL} / \mathrm{g})$ & $\alpha_{0} \times 10^{4}\left({ }^{\circ} C^{-1}\right)$ & $b_{0}\left(\mathrm{MPa}^{-1}\right)$ & $b_{1}\left({ }^{\circ} C^{-1}\right)$ & $\gamma^{b}$ \\
\hline 0 & 0.8431 & 0.8290 & 7.86 & 257 & 0.0083 & $2.21 \pm 0.14$ \\
\hline 1.8 & 0.8305 & 0.8190 & 6.98 & 276 & 0.0087 & $2.27 \pm 0.04$ \\
\hline 4.3 & 0.8141 & 0.8061 & 6.76 & 244 & 0.0060 & $2.37 \pm 0.05$ \\
\hline 8.2 & 0.7942 & 0.7833 & 6.87 & 248 & 0.0059 & $2.80 \pm 0.17$ \\
\hline 11.9 & 0.7729 & 0.7569 & 6.76 & 383 & 0.0128 & $3.30 \pm 0.10$ \\
\hline 15.3 & 0.7533 & 0.7441 & 6.06 & 270 & 0.0057 & $3.39 \pm 0.11$ \\
\hline 20.5 & 0.7253 & 0.7162 & 5.69 & 332 & 0.0083 & $3.70 \pm 0.20$ \\
\hline 28.2 & 0.6884 & 0.6827 & 4.80 & 330 & 0.0064 & $3.78 \pm 0.17$ \\
\hline
\end{tabular}

${ }^{a}$ At ambient temperature and pressure. ${ }^{b}$ Calculated using the specific volume after subtraction of the silica content.

temperatures the peaks for the two compositions superpose, apart from the rise toward lower frequencies due to dc conductivity (transport of mobile ions). This shows that the silica does not alter the segmental dynamics.

In Figure 12, the segmental relaxation times, defined as $\tau_{\alpha}$ $=1 /\left(2 \pi f_{\max }\right)$, where $f_{\max }$ is the frequency of the maximum in the dielectric loss, are plotted vs reciprocal temperature. The $\tau_{\alpha}(T)$ of the unfilled and filled PVAc are equivalent and the non-Arrhenius behavior can be described by the Vogel-Fulcher equation 56

$$
\log \tau_{\alpha}=-11.72+\frac{611}{T-265.7}
$$

where $T$ is in kelvin. At the calorimetric $T_{\mathrm{g}}, \tau_{\alpha}=11 \mathrm{~s}$.

Bulk Modulus. Although the PVT data illustrated in Figure 7 can be used to determine the bulk modulus, it is more accurate to employ isothermal measurements for this purpose. Accordingly, isothermal specific volumes were measured as a function of pressure at various fixed temperatures above $T_{\mathrm{g}}$ and fit to the Tait equation of state ${ }^{57}$

$V(T, P)=V_{0} \exp \left(-\alpha_{0} T\right)\{1-0.0894 \ln [1+P / B(T)]\}$

where $\alpha_{0}$ is the thermal expansion coefficient at zero pressure

$$
B(T)=b_{0} \exp \left(-b_{1} T\right)
$$

and $V_{0}, b_{0}$, and $b_{1}$ are material constants. Note that over the limited range of the measurements $\alpha_{P}$ was constant within either the glassy or rubbery states. The Tait parameters for each composition are listed in Table 1; these also give a good description of the isobaric $P V T$ data measured for the rubbery state. From eq 6 the bulk modulus is calculated as ${ }^{58}$

$$
K=\frac{(B+P) V}{0.0894 V_{0}}
$$

with results shown in Figure 13. The bulk modulus increases with silica content, and since the bulk modulus of silica is very large ( $\sim 30$-fold larger than $K$ of neat PVAc),${ }^{59}$ there is negligible error in assuming that the compressibility arises solely from the polymer. With this assumption the $\phi$ dependence of $K$ is accounted for entirely by the amount of polymer in the composition (solid line in Figure 13). Note that assuming the occluded polymer is incompressible would yield calculated values of $K$ (dashed line) significantly larger than the measured bulk modulus.

\section{Discussion}

An outstanding issue in filler reinforcement is the nature of the polymer proximate to the particle surface. There have been various reports of constraints from the filler causing vitrification of the interfacial chains, ${ }^{13-24}$ including recent works ${ }^{42,43}$ in which silica particles were reported to affect the glass transition of the polymer. However, other studies found no evidence of such an effect. ${ }^{23,24,51}$ From $\eta(\phi)$ and eq 1 we deduce that as much as $10 \%$ of the polymer is isolated from the shear field at the higher silica concentrations. The quantity of this occluded rubber is close to the bound rubber content (Figure 3). Nevertheless, this shielding of the interfacial polymer does not alter $T_{\mathrm{g}}$, which is constant $\left(=40.4 \pm 0.7^{\circ} \mathrm{C}\right)$ for all compositions. Moreover, the segmental relaxation dispersion and relaxation times show no evidence of immobilized polymer. Consistent with these results, the magnitude of the changes in heat capacity and thermal expansivity at $T_{\mathrm{g}}$ are strictly proportional to the polymer concentration; that is, the diminution in $\Delta C_{P}$ or $\Delta \alpha_{P}$ with $\phi$ can be quantitatively accounted for without invoking the presence of constrained polymer, in the manner necessary to describe the viscosity data. None of the experiments carried out herein provide evidence of any suppression of the segmental mobility of PVAc chains residing at the interface with the silica.

Although the local segmental relaxation function (Figure 11) and relaxation times (Figure 12) are unaffected by the presence of silica nanoparticles, the dielectric spectra for the filled and unfilled material are not identical. In Figure 11 the rise toward lower frequencies, due to the contribution to the dielectric loss from mobile charge carriers, is larger for $\phi>0$. This contribution has a frequency dependence given by

$$
\epsilon_{\mathrm{dc}}^{\prime \prime}=\sigma_{\mathrm{dc}} /\left(\omega \epsilon_{0}\right)
$$

in which $\sigma_{\mathrm{dc}}$ is the (frequency independent) dc conductivity and the vacuum permittivity $\epsilon_{0}=8.854 \times 10^{-12} \mathrm{~F} / \mathrm{m}$. The magnitude of $\sigma_{\mathrm{dc}}$ depends on both the concentration of ions and their mobility. According to the Debye-Stokes-Einstein relation, ${ }^{60}$ the segmental mobility governs the ion mobility. This means that the higher $\sigma_{\mathrm{dc}}$ for the filled PVAc must reflect the presence of more ions, presumably associated with the tetrasulfidosilanetreated particle surface. In Figure 12, the $\tau_{\alpha}$ are seen to have a somewhat steeper slope than the curves for $\sigma_{\mathrm{dc}}$. This underlies the smaller separation of the conductivity and the relaxation peak at the lower temperature in Figure 11. This is a well-known effect usually ascribed to decoupling of translational and orientational motions; ${ }^{55}$ however, it can also arise if the ion concentration varies with temperature. ${ }^{61}$

PVAc in the rubbery state has a thermal expansivity and bulk compressibility much larger than those of silica (by factors of roughly 800 and 30, respectively), and we find that the $\phi$ dependence of $\alpha_{P}$ and $K$ can be predicted from the values for the neat PVAc. An important implication is that neither the occluded rubber nor the formation of a filler network exerts any influence on these properties. The latter is true even though the associated volumetric strains are quite small $(<1 \%$ for the data in Figure 13) and below the shear strains associated with disruption of the network (Figure 4). The percolation threshold for network formation (i.e., continuity of particle contacts extending from one side to the other), $\phi \sim 0.11$ herein (Figure 
5 ), is within the usual range for rigid fillers in soft polymers, i.e., $6-35 \mathrm{vol} \%$ depending on the size and shape of the particles and their interaction with the polymer. ${ }^{62}$ We can also conclude that the interaction of the PVAc with the coated silica is sufficient to prevent debonding and vacuole formation, which otherwise could give rise to anomalies in the $V(P)$ response.

A surprising result herein is that the volume sensitivity of the segmental dynamics increases systematically with silica content, notwithstanding the absence of changes in glass transition properties, such as $T_{\mathrm{g}}, \tau_{\alpha}, \Delta C_{P}$, and $\Delta \alpha_{P}$. The effect of temperature on $\tau_{\alpha}$ is invariant to the presence of the silica particles, even though the latter change the volume dependence of $\tau_{\alpha}$. The scaling exponent $\gamma$ increases from 2.2 to 3.8 over the range from $\phi=0$ to $28.2 \%$. To put this in perspective, we can calculate the ratio of the isochoric activation energy, $E_{V}=$ $\left.R\left[(\partial \ln \tau) / \partial T^{-1}\right]\right|_{V}$, to the isobaric activation enthalpy, $H_{P}=R[(\partial$ $\left.\ln \tau) / \partial T^{-1}\right]\left.\right|_{P}$, using the relation ${ }^{63}$

$$
\frac{E_{V}}{H_{P}}=\left(1+\alpha_{P} T \gamma\right)^{-1}
$$

The quantity $E_{V} / H_{P}$ quantifies the relative effect of temperature and volume to the segmental dynamics, ${ }^{64}$ varying from zero for volume-dominated dynamics to unity for segmental relaxation governed strictly by thermal energy. Inserting in eq 10 the thermal expansion coefficient for PVAc, we obtain at $T_{\mathrm{g}}$ $E_{V} / H_{P}=0.77$ for neat PVAc, decreasing to 0.69 at the highest silica levels. These values are toward the high end of the range found for polymers, $0.25 \leq E_{V} / E_{P} \leq 0.81,{ }^{55}$ and being larger than 0.5 indicate that temperature is a stronger control variable than volume. The decrease in $E_{V} / E_{P}$ with silica content is consistent with the report of silica particles increasing the unoccupied volume of a polymer, ${ }^{39}$ noting that this ratio also increases with temperature. ${ }^{65}$ If the local dynamics become more sensitive to volume in the presence of reinforcing filler, the implication is that properties dominated by volume will be especially affected by fillers. One such property is physical aging (or "structural relaxation"), which is known to be influenced by interfacial constraints. ${ }^{66-69} \mathrm{We}$ are currently carrying out experiments to investigate this aspect of particle-reinforced polymers.

\section{Summary}

The main conclusions of this work are the following:

1. PVAc containing silica nanoparticles exhibits the classic mechanical behavior of filler-reinforced rubber. At silica levels $\geq 11$ vol \%, the dynamic storage modulus decreases with strain amplitude due to disruption of the agglomerated particle network (Payne effect). The quantity of the colloidal silica required to form a network falls within the range for small particle, high structure carbon black.

2. As judged by the composition dependence of the viscosity, there is a substantial quantity of the polymer shielded from shear stresses, leading to large increments in $\eta$ with silica content. Except for low levels of silica, this occluded rubber is less than the bound rubber, indicating that not all chains tethered to silica particles are screened from the flow.

3. Notwithstanding the effect of the occluded interfacial chains on the flow properties, the various manifestations of the glass transition, including the (smeared) jumps in heat capacity, thermal expansivity, and bulk modulus, as well as $T_{\mathrm{g}}$ itself, give no evidence of any constrained boundary layer; that is, the interfacial PVAc behaves no differently from the bulk material. Moreover, the local segmental relaxation process, which un- derlies the glass transition, is unaffected by the presence of silica particles. The only effect of the silica on the glass transition is to weaken its intensity through reduction in the polymer concentration.

4. In the rubbery state above $T_{\mathrm{g}}$, the thermal expansion coefficient and bulk modulus depend only on the quantity of PVAc present; the contribution of the silica or occluded rubber is negligible. This is consistent with the very low $\alpha_{P}$ and large $K$ of the glassy, networked $\mathrm{SiO}_{2}$ particles and with the absence of interfacial effects on the polymer.

5. Although the silica does not measurably influence the properties at the glass transition, including the magnitude and temperature dependence of the segmental relaxation time, the segmental dynamics exhibit greater volume sensitivity with increasing silica. This is reflected in the magnitude of the scaling exponent, which increases from $\gamma=2.2$ for neat PVAc to a value of 3.8 for $\phi=0.28$. There is an abrupt change in $\gamma$ at the concentration of silica for which the Payne effect is manifested in the mechanical measurements.

Acknowledgment. The work at the Naval Research Laboratory was supported by the Office of Naval Research. C.G.R. and A.M.R. thank Bridgestone Americas for permission to publish. R.B. acknowledges the American Society for Engineering Education for a postdoctoral fellowship.

\section{References and Notes}

(1) Payne, A.; Whittaker, R. Rubber Chem. Technol. 1971, 44, 440.

(2) Medalia, A. I. Rubber Chem. Technol. 1974, 47, 437; 1978, 51, 411.

(3) Roland, C. M.; Robertson, C. G.; Nikiel, L.; Puskas, J. E. Rubber Chem. Technol. 2004, 77, 372

(4) Zhu, Z. Y.; Thompson, T.; Wang, S. Q.; von Meerwall, E. D.; Halasa, A. Macromolecules 2005, 38, 8816.

(5) Goritz, D.; Raab, H.; Frohlich, J.; Maier, P. G. Rubber Chem. Technol. 1999, 72, 929.

(6) Robertson, C. G.; Wang, X. Europhys. Lett. 2006, 76, 278.

(7) Robertson, C. G.; Wang, X. Phys. Rev. Lett. 2005, 95, 075703.

(8) Wang, X.; Robertson, C. G. Phys. Rev. E 2005, 72, 031406.

(9) Robertson, C. G.; Bogoslovov, R.; Roland, C. M. Phys. Rev. E 2007, 75,051403 .

(10) Caggioni, M.; Spicer, P. T.; Blair, D. L.; Lindberg, S. E.; Weitz, D. A. J. Rheol. 2007, 51, 851.

(11) Bogoslovov, R.; Roland, C. M.; Gamache, R. M. Appl. Phys. Lett. 2007, 90, 221910

(12) Doi, M.; Edwards, S. F. The Theory of Polymer Dynamics; Clarendon: Oxford, 1986.

(13) Montes, H.; Lequeux, F.; Berriot, J. Macromolecules 2003, 36, 8107.

(14) Struik, L. C. E. Polymer 1987, 28, 1534.

(15) Wang, M.-J. Rubber Chem. Technol. 1998, 71, 520

(16) Berriot, J.; Montes, H.; Lequeux, F.; Long, D.; Sotta, P. Macromolecules 2002, 35, 9756.

(17) Kader, M. A.; Kim, K.; Lee, Y.-S.; Nah, C. J. Mater. Sci. 2006, 41 , 7341

(18) Iqbal, A.; Frormann, L.; Saleem, A.; Ishaq, M. Polym. Compos. 2007, $28,186$.

(19) Lopez-Martinez, E. I.; Marquez-Lucero, A.; Hernandez-Escobar, C. A.; Flores-Gallardo, S. G.; Ibarra-Gomez, R.; Yacaman, M. J.; Zaragoza-Contreras, E. A. J. Polym. Sci., Polym. Phys. Ed. 2007, 45, 511.

(20) Aras, L.; Sheldon, R. P.; Lai, H. M. J. Polym. Sci., Polym. Phys. Lett. 1978, 16, 27.

(21) Bandi, S.; Schiraldi, D. A. Macromolecules 2006, 39, 6537.

(22) Kirst, K. U.; Kremer, F.; Litvinov, V. M. Macromolecules 1993, 26 , 975.

(23) Leyva, M. E.; Barra, G. M. O.; Moreira, A. C. F.; Soares, B. G.; Khastgir, D. J. Polym. Sci., Polym. Phys. Ed. 2003, 41, 2983.

(24) Kraus, G.; Gruver, J. T. J. Polym. Sci., Part A 1970, 8, 571.

(25) Roth, C. B.; Dutcher, J. R. J. Electroanal. Chem. 2005, 584, 13.

(26) Grohens, Y.; Hamon, L.; Reiter, G.; Soldera, A.; Hol, Y. Eur. Phys. J. E 2002, 8, 217.

(27) Alcoutlabi, M.; McKennam, G. B. J. Phys.: Condens. Matter 2005 , 17, R461.

(28) Mijovic, J.; Lee, H. K.; Kenny, J.; Mays, J. Macromolecules 2006 , 39, 2172. 
(29) Lee, Y. H.; Bur, A. J.; Roth, S. C.; Start, P. R. Macromolecules 2005, 38,3828

(30) Hoang, G. C.; Nikiel, L.; Gerspacher, M. J. Korean Phys. Soc. 2004, 44, 962.

(31) Roland, C. M.; Lee, G. F. Rubber Chem. Technol. 1990, 63, 554.

(32) Byers, J. T. Rubber Chem. Technol. 2002, 75, 527.

(33) Wang, M.-J.; Tu, H.; Murphy, L.; Mahmud, K. Rubber Chem. Technol. 2000, 73, 666

(34) Hu, H.; Lin, J.; Zheng, Q.; Xu, X. J. Appl. Polym. Sci. 2006, 99, 3477

(35) Winberg, P.; Desitter, K.; Dotremont, C.; Mullens, S.; Vankelecom, I. F. J.; Maurer, F. H. J. Macromolecules 2005, 38, 3776.

(36) Hanna, F. F.; Bishai, A. M.; Ward, A. A.; Stoll, B.; Goritz, D. Kaut. Gummi Kunst. 2004, 57, 288.

(37) Maxwell, R. S.; Chinn, S. C.; Solyom, D.; Cohenour, R. Macromolecules 2005, 38, 7026.

(38) Brown, D.; Mele, P.; Marceau, S.; Alberola, N. D. Macromolecules 2003, 36, 1395.

(39) Merkel, T. C.; Toy, L. G.; Andrady, A. L.; Gracz, H.; Stejskal, E. O Macromolecules 2003, 36, 353.

(40) Sternstein, S. S.; Zhu, A. J. Macromolecules 2002, 35, 7262.

(41) Fragiakakis, D.; Pissis, P.; Bokobza, L. J. Non-Cryst. Solids 2006. 352, 4969.

(42) Fragiakakis, D.; Pissis, P. J. Non-Cryst. Solids 2007, 353, 4344.

(43) Sargsyan, A.; Tonoyan, A.; Davtyan, S.; Schick, C. Eur. Polym. J. 2007, 43, 3113.

(44) Zoller, P.; Walsh, D. J. Standard Pressure-Volume-Temperature Data for Polymers; Technomic: Lancaster, PA, 1995.

(45) Medalia, A. I. Rubber Chem. Technol. 1972, 45, 1171; 1973, 46, 877.

(46) Guth, E. J. Appl. Phys. 1945, 16, 20.

(47) Quemada, D. Rheol. Acta 1977, 16, 82 and references therein.

(48) Oliver, D. R.; Ward, S. G. Nature (London) 1953, 171, 396.

(49) Roland, C. M.; Peng, K. L. Rubber Chem. Technol. 1991, 64, 790

(50) Roland, C. M.; Robertson, C. G.; Nikiel, L.; Puskas, J. E. Rubber Chem. Technol. 2004, 77, 372.
(51) Robertson, C. G.; Lin, C. J.; Rackaitis, M.; Roland, C. M. Macromolecules, in press.

(52) Lord, R. C.; Morrow, J. C. J. Chem. Phys. 1957, 26, 230.

(53) Nikiel, L.; Yang, H.; Gerspacher, M.; Hoang, G. Kaut. Gummi Kunst. 2004, 57, 538

(54) White, G. K. J. Appl. Phys. 1973, 6, 2070.

(55) Roland, C. M.; Hensel-Bielowka, S.; Paluch, M.; Casalini, R. Rep. Prog. Phys. 2005, 68, 1405.

(56) Broadband Dielectric Spectroscopy; Kremer, F., Schonhals, A., Eds.; Springer-Verlag: Berlin, 2003.

(57) Orwoll, R. A. In Physical Properties of Polymers Handbook; Mark, J. E., Ed.; AIP Press: Melville, NY, 1996.

(58) Hartmann, B.; Yee, G. F.; Balizer, E. J. Acoust. Soc. Am. 2000, 108 65.

(59) Huang, L.; Duffrene, L.; Kieffer, J. J. Non-Cryst. Solids 2004, 349, 1.

(60) Hansen, J. P.; McDonald, I. R. Theory of Simple Liquids, 2nd ed.; Academic Press: London, 1986.

(61) Power, G.; Vij, J. K.; Johari, G. P. J. Phys. Chem. B 2007, 111, 11201

(62) Chung, K. T.; Sabo, A.; Pica, A. P. J. Appl. Phys. 1982, 53, 6867

(63) Casalini, R.; Roland, C. M. Phys. Rev. E 2004, 69, 062501.

(64) Williams, G. In Dielectric Spectroscopy of Polymeric Materials; Runt, J. P., Fitzgerald, J. J., Eds.; American Chemical Society: Washington, DC, 1997.

(65) Roland, C. M.; McGrath, K. J.; Casalini, R. J. Non-Cryst. Solids 2006, 352,4920

(66) Lu, H. B.; Nutt, S. Macromolecules 2003, 36, 4010.

(67) Lee, A.; Lichtenhan, J. D. Macromolecules 1998, 31, 4970.

(68) Rittigstein, P.; Torkelson, J. M. J. Polym. Sci., Polym. Phys. Ed. 2006, 44, 2935.

(69) Priestley, R. D.; Rittigstein, P.; Broadbelt, L. J.; Fukao, K.; Torkelson, J. M. J. Phys.: Condens. Matter 2007, 19, 205120. 